\title{
DIRECTIONAL REASSEMBLY OF MYOFIBRILS IN CULTURED CARDIAC MYOCYTES USING A THREE-DIMENSIONAL POLYMERIC SUBSTRATE
}

\author{
Yi Zhao, and Xin Zhang \\ Laboratory for Microsystems Technology \\ Department of Manufacturing Engineering, Boston University \\ Boston, MA 02215, USA
}

\begin{abstract}
This paper presents directional reassembly of myofibrils in cardiac myocytes using a three-dimensional polymeric substrate. Such substrate modulates reorganization of dismantled myofibrils through microstructures, resembling the morphology in vivo. As a result, the cardiac myocytes in culture are reoriented. The mechanical forces at subcellular level are in situ monitored during the adhesion and redifferentiation processes using microfabricated polymeric structures, revealing evolution of the mechanical interaction between cardiac myocytes in culture and the artificial extracellular environment. This polymeric substrate has the potential to be a basis for development of in vitro assembled cardiac tissue.
\end{abstract}

\section{INTRODUCTION}

Heart is the most heavily worked organ in the body, pumping blood to and from all the other organs. The contractile performance of the heart heavily depends on the mechanical behavior on cellular and subcellular level. Specifically, the myofibrils within the cardiac myocytes have a directional arrangement, allowing the cells to perform large contraction and to pump as much blood as required. However, such contractile performance is somewhat deteriorated in cultured myocytes due to remodeling [1]. Many mechanical properties in vivo disappear because of the randomly oriented myofibrils. Therefore, there is a need to reassemble the myofibrils in a directional profile with a hope to resemble the contractile performance in vivo, facilitating the cardiomechanical study and cardiac tissue development.

Prior research in tissue engineering has shown that the microfabricated structures have potentials in regulation of a wide variety of cellular behaviors, such as cell growth and attachment [2, 3]. The cells cultured on such structures adapt to the artificial extracellular environment and adjust their physiologic performance accordingly. In this paper, we report the use of a polymeric substrate with three-dimensional microstructures in regulation of the reassembly of myofibrils during cell remodeling process. The cellular mechanical forces are in situ monitored during the adhesion and redifferentiation process to provide evidence of directional myofibrils reorganization.

\section{FABRICATION AND SETUP}

The three-dimensional substrate (Figure 1) is made of PDMS polymer. It consists of parallel sidewalls, embedded pillars and large polymeric posts. The distance between adjacent sidewalls is about $20 \mu \mathrm{m}$, in accordance with the width of a single cardiac myocyte. In the spaces between the parallel sidewalls, large polymeric posts are placed, being $100 \mu \mathrm{m}$ to $150 \mu \mathrm{m}$ away from one another. These posts are used to separate individual myocytes so that the cells will not interconnect. The embedded pillars are 2 $\mu \mathrm{m}$ in diameter and closely spaced, serving as mechanical sensors for force measurement. In our design, the PDMS sidewalls and the large posts are slightly higher than the embedded pillars so as to form a recessed chamber for the cells. Figure 1c shows the schematics of cell attachment onto the substrate.
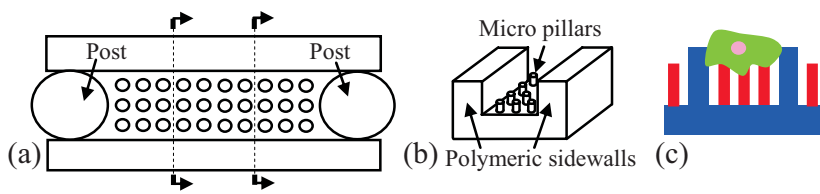

Figure 1. The three-dimensional PDMS substrate: (a), (b) the substrate is made up of polymeric posts, parallel sidewalls and embedded pillars; (c) the cell attachment on the substrate.

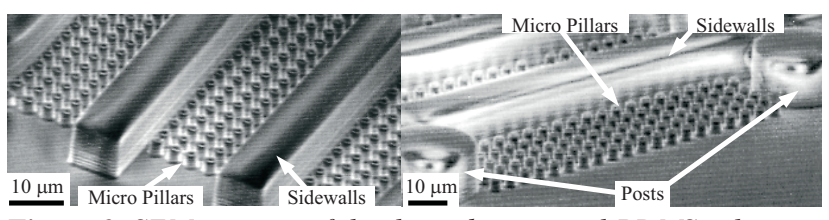

Figure 2. SEM pictures of the three-dimensional PDMS substrate with microstructures of different heights.

This three-dimensional polymeric substrate was fabricated using pressure-assisted micro molding technology we reported earlier [4]. The microstructures with different heights were fabricated via one replication step using single-crystal silicon template. The fabricated substrate is shown in Figure 2. The substrate was placed in an on-stage culture chamber and integrated with an inverted optical microscope. The fluidic and electrical connections were supplied to ensure the cells were in a preferable environment (i.e. $37^{\circ} \mathrm{C}$ and $5 \% \mathrm{CO}_{2} /$ air).

\section{MYOFIBRIL REASSEMBLY \& CELL ALIGNMENT}

Left ventricular myocytes were isolated from male Wistar rats according to a previously described protocol [5] and cultured on the three-dimensional substrate. The cell morphology change during the adhesion and redifferentiation processes was monitored. The cellular forces were measured from the embedded polymeric pillars.

The morphology change is illustrated in Figure 3. During the first 24 hours, the cells retained rod-shaped morphology as in vivo. In the following few days, the myofibrils were dismantled. As a result, the cells lost their striated architecture and transformed to an amorphous form. At about 72 hours, most cells were rounded. Therefore, there were no substantial cellular forces measured at this moment. As the culture continued, myofibrils reorganized and the cells stretched out pseudopodia towards the substrate. In the plain substrate, the pseudopodia proceeded in all directions so that the cells spread isotropically. The three-dimensional polymeric substrate, on the other hand, acted as scaffolds to guide the myofibrillar proteins along the longitudinal axis of the sidewalls, thus reorienting the cells.

The cell reorientation after 7 days culture is shown in Figure 4. It is seen from the fluorescence labeled actin filaments that the cells were well confined by the microfabricated sidewalls and being aligned by the three-dimensional structures. This is in sharp contrast to the control group where the cells were cultured on a plain polymeric substrate. A closer observation shows the myofibrils reassembly in aligned cells had a directional morphology. In the control group, however, the myofibril reassembly was randomly oriented. 


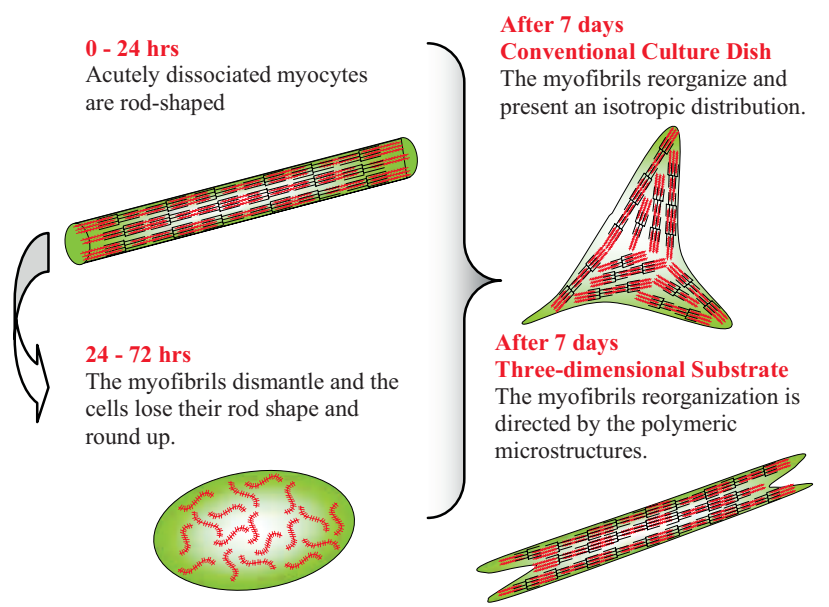

Figure 3. Remodeling process of cardiac myocytes in long-term culture. The reassembling of myofibril adapts to the local extracellular environment.

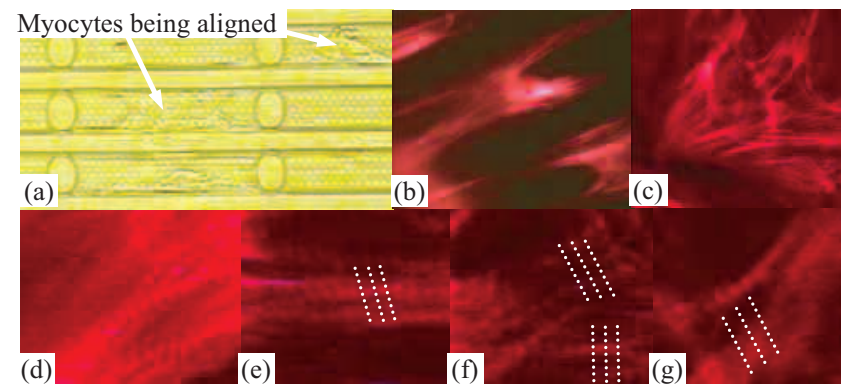

Figure 4. The reorientation of myocytes is observed by (a) the phase contrast image and (b) fluorescence labeling of the actin filaments. (c) shows the control group cultured on plain PDMS surface. A closer observation shows the reassembling of myofibrils is directional in (d) the aligned cells and randomly oriented in $(e)-(g)$ the control group.

\section{FORCES DURING REDIFFERENTIATION}

With reassembly of the myofibrils and reorientation of the cells, contractile performance of the cardiac myocytes resumes. Given the fact that the large polymeric posts eliminate the physical contacts between neighboring cells, the functional gap junction is not present, as evidenced by the lack of synchronous contraction. The cell contraction is therefore driven by each cell's own pacemaker. The mechanical forces generated by the cells were determined from deformation of free ends of the micro pillars.

Figure 5 shows an aligned myocyte and the subcellular mechanical force during its contraction. The deformation of the pillars was monitored by a video camera. The force vector map was achieved by multiplying the deformation with the predetermined spring constant, showing a subcellular spatial resolution. The magnitude and direction of the cellular forces at the locations of different pillars were directly achieved from the force map. Furthermore, by assuming the cellular forces have a spatially continuous distribution, data interpolation was conducted so that the cellular forces at the locations other than the pillars were approximated. As can be seen from the force map, the major force difference between the diastolic and systolic states is along longitudinal axis of the polymeric sidewall, providing evidence of directional reassembly of the myofibrils along this direction. Therefore, the force measurement during the redifferentiation process validates this three-dimensional polymeric substrate a viable tool for reassembling the myofibrils directionally.

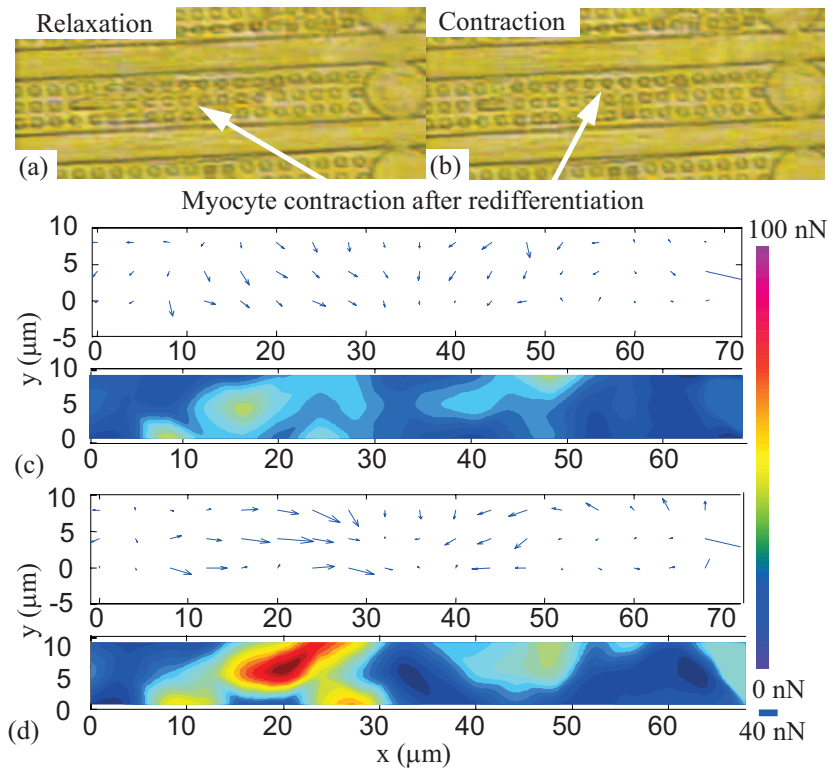

Figure 5. (a)-(b): The contractile performance of the cells reappears when the myofibrils reassemble. The cellular forces at (c) the diastolic state and (d) the systolic state are derived from the deformation of the micro pillars.

\section{CONCLUSION}

This study reports reorientation of myofibrils and alignment of cultured caridiac myocytes by using a polymeric substrate. The three-dimensional microstructures were built on the substrate via pressure-assisted micro molding. Long-term culture showed that the polymeric substrate can successfully modulate the reorganization of the myofibrils during the cell redifferentiation, thus aligning the myocytes along specific direction. The directional reorganization of myofibrils was confirmed by monitoring the mechanical forces developed from the aligned cells. This work promises the potential for in vitro assembled cardiac tissue and can lead to less sacrifice of time, cost and animal donors.

\section{ACKNOWLEDGEMENTS}

This work is supported by the NSF CAREER Award. The authors would like to thank Professor Chee Chew Lim, Professor Douglas B. Sawyer at Boston University School of Medicine, and Professor Ronglih Liao at Harvard Medical School for their technical advices.

\section{REFERENCES}

1. A.C. Nag, M.L. Lee, F.H. Sarkar, "Remodelling of adult cardiac muscle cells in culture: dynamic process of disorganization and reorganization of myofibrils", J. Muscle. Res. Cell. Motil. 17(3), 313 (1996).

2. A. Folch, S. Mezzour, M. During, O. Hurtado, M. Toner, R. Muller, "Stacks of Microfabricated Structures as Scaffolds for Cell Culture and Tissue Engineering", Biomedical Microdevices, 2(3):207, (2000).

3. S.N. Bhatia and C.S. Chen, "Tissue Engineering at the MicroScale", Biomedical Microdevices, 2(2):131, (1999).

4. Y. Zhao and X. Zhang, "Adaptation of flexible polymer fabrication to cellular mechanics study", Appl. Phys. Lett., 87 (14): 144101, (2005).

5. C.C. Lim, M.H. Helmes, D.B. Sawyer, M. Jain, R. Liao, "Highthroughput assessment of calcium sensitivity in skinned cardiac myocytes", Am. J. Physiol. Heart Circ. Physiol. 281: H969 (2001). 